\title{
Bilateral Inguinal Lymphadenopathy as Initial Manifestation of Sarcoidosis
}

\author{
Samer Makki Mohamed Al-Hakkak \\ Department of Surgery, Faculty of Medicine, Jabir Ibn Hayyan Medical University, Najaf City, Iraq.
}

\section{Corresponding Author:}

Dr. Samer Makki Mohamed Al-Hakkak

Email: sammerhakak1971@yahoo.com

This is an Open Access article distributed under the terms of the Creative Commons Attribution License (creativecommons.org/ licenses/by/3.0).

Received : March 19, 2017

Accepted : May 30, 2017

Published : June 25, 2017

\begin{abstract}
Background: Sarcoidosis is a systemic granulomatous disorder of unknown cause characterized by frequent pulmonary involvement. Despite pulmonary involvement in most patients, sarcoidosis can have varied presentations. The clinical presentation of sarcoidosis is highly variable. Lymph node involvement is rarely found in isolation. Case Report: A 57-year-old asymptomatic male presented with bilateral pelvic and inguinal lymphadenopathy. A computed tomography (CT) scan of the chest, abdomen, and pelvis revealed bilateral deep pelvic and inguinal bulky adenopathy. Excision biopsy revealed sarcoidosis. He responded to six months of oral steroid treatment. Followup computed tomography scan after 6 months of steroid therapy showed complete resolution. Conclusion: This case exemplifies the difficulty in diagnosing sarcoidosis in its initial stages. A high index of suspicion and excision biopsy is the key in diagnosing this condition.
\end{abstract}

Keywords: Abdomen, Biopsy, Sarcoidosis, Lymph nodes, Lymphadenopathy.

\section{Introduction}

Sarcoidosis is an inflammatory disease with varied presentations thus leading to confusion whether asymptomatic individuals with suspected diagnosis of sarcoidosis should be investigated further. The clinical presentation of sarcoidosis varies from complicated and severe to uncomplicated and mild to asymptomatic sarcoidosis. A substantial percentage of sarcoidosis-affected individuals may never manifest clinical disease and upto $30 \%$ have spontaneous remission $[1,2]$. Diagnosis of asymptomatic presentations in sarcoidosis remains a challenge as it can influence prognosis. The presentation of sarcoidosis depends on epidemiological factors such as age, sex, and race, the duration of the disease and the sites of involvement. Overall, sarcoidosis is mostly revealed in the following circumstances: (i) respiratory symptoms, firstly persistent dry cough in around 30\% cases, (ii) extra-thoracic localizations, mainly peripheral lymph nodes, eyes or skin, (iii) constitutional symptoms such as fatigue (27\%), weight loss $(28 \%)$, fever (10-17\%) or night sweats and (iv) erythema nodosum (3-44\%) [3,4,5]. Constitutional symptoms, particularly asthenia, are often present and can be a disabling problem [6]. Finally, an incidental discovery of sarcoidosis in asymptomatic patients with chest X-ray aberrations is also not uncommon (8-60\%) [7].

\section{Case Report}

A 57-year-old non-insulin dependent diabetic male was referred to general surgical clinic from urologist where he was being treated for benign prostatic hypertrophy. Urologist incidentally discovered bilateral inguinal lymph nodes and sent him to the surgeon for further assessment. On examination, there were painless multiple bilateral inguinal swellings of size about $4 \times 3 \mathrm{~cm}$ without any skin changes, erythema or tenderness. Axillary and cervical lymph nodes were not palpable. No significant findings were noted on abdominal, anal, 
genital and lower limb examination. Routine blood investigations revealed hemoglobin $10.5 \mathrm{~g} / \mathrm{dL}$ (13-15), total leukocyte count 4600 cells $/ \mu \mathrm{L}$ (4000-11000), platelet counts 330000 cells $/ \mu \mathrm{L}$, an erythrocyte sedimentation rate of $47 \mathrm{~mm} /$ hour (0-22), and C-reactive protein level of $4.8 \mathrm{mg} / \mathrm{dL}$ $(<3 \mathrm{mg} / \mathrm{dL})$. Ultrasound of abdomen revealed bilateral multiple pelvic lymphadenopathies, the largest on right side measuring $4 \times 3 \mathrm{~cm}$, and about $3.2 \times 3 \mathrm{~cm}$ on left side. Ultrasound also showed bilateral multiple matted inguinal lymph nodes with largest lymph node about $4.2 \times 2 \mathrm{~cm}$. A computed tomography (CT) scan of the chest, abdomen, and pelvis revealed bilateral deep pelvic and inguinal bulky adenopathy, with $6 \times 3 \mathrm{~cm}$ both pelvic sidewall mass, multiple enlarged bilateral inguinal lymph nodes measuring $4 \mathrm{~cm}$. No abnormalities were noted in chest or abdomen. Excisional biopsy left inguinal lymph node was taken. The macroscopic finding showed, single large lymph node, $4.5 \times 3 \times 1.5 \mathrm{~cm}$ partly bisected, the cut surfaces showing white homogeneous areas [Fig.1a,1b]. Microscopy showed the destruction of lymphoid architecture by multiple caseating granulomas with a presence of multinucleated giant cells with the area of calcification (Shaumann bodies). The finding of chronic granulomatous inflammation was consistent with sarcoidosis [Fig.2a,2b]. The patient was treated with prednisolone tablets 40 $\mathrm{mg}$ daily for two weeks followed by $20 \mathrm{mg}$ daily after four months. Multiple ultrasounds of his abdomen and inguinal region showed decrease in lymph nodes size. A repeated CT scan of his chest, abdomen, and pelvis 5 months after his initial diagnosis suggested satisfactory progress of sarcoidosis.

\section{Discussion}

Sarcoidosis is the ubiquitous disease of unknown etiology, seen worldwide with a multisystem involvement [3]. Early diagnosis is the key to prevent the potentially damaging effects of sarcoidosis. There is no laboratory test that will diagnose sarcoidosis for certain. Sarcoidosis is
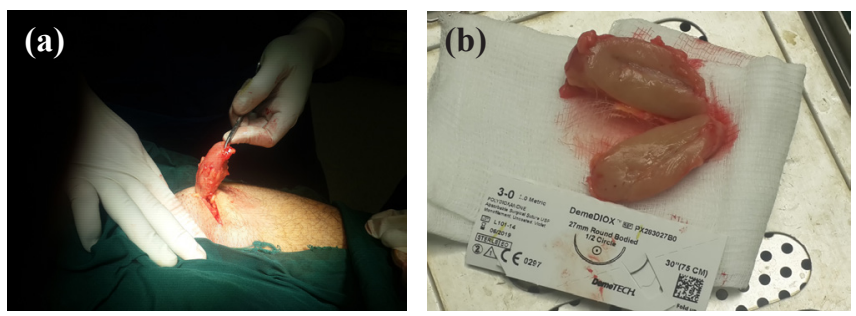

Fig.1(a): Open biopsy from the left inguinal lymph node. (b): Bisected lymph node: homogenous, white in color.
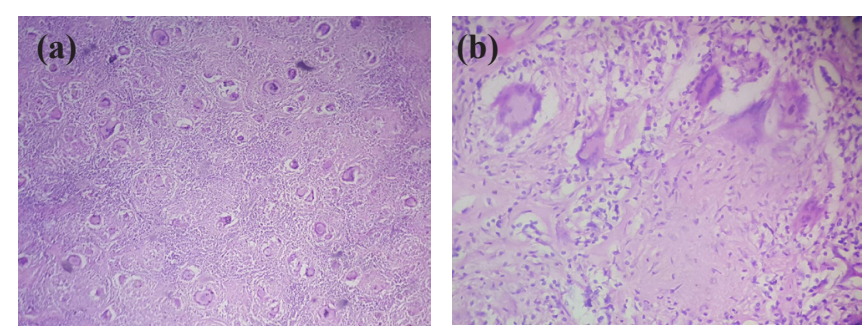

Fig.2(a): Low power field microscopy of lymph node biopsy showing a granuloma without central necrosis, suggestive of sarcoidosis (H\& E stain, 40X). (b): Non-caseating granuloma with a presence of multinucleated giant cells with the area of calcification (Schaumann bodies) ( $H \&$ E stain, 100X).

diagnostic dilemma due to an absence of precise consistent case definition, variability in clinical presentation and lack of confirmatory diagnostic tests.

Many people with sarcoidosis may not require any treatment, and self-resolution of sarcoidosis might occur after several years without causing serious complications. The diagnosis of sarcoidosis is established on the basis of compatible clinical and radiologic findings, supported by histologic evidence in one or more organs of noncaseating epithelioid-cell granulomas. Isolated peripheral lymphadenopathy is the uncommon presentation, particularly involving the cervical, axillary, epitrochlear, and inguinal nodes [4]. Important differential diagnoses of granulomatous disease like Crohn's disease, lymphoma, and vacuities should be excluded. In our case, inguinal lymph node biopsy showed caseating granuloma. The appropriate therapy for sarcoidosis has not yet been well defined for all patients $[9,10,11]$. Between 30 and $70 \%$ of patients never require therapy. In 
the other cases, a treatment, often corticosteroids, is necessary, either at the onset of the disease or during follow-up because of various consequences.

\section{Conclusion}

Sarcoidosis should be considered as a part of differential diagnosis in asymptomatic inguinal lymphadenopathy.

Contributors: SMMA wrote the manuscript, did literature search, will act as guarantor and approved final version of the manuscript.

Funding: None; Competing interests: None stated.

\section{References}

1. George J, Graves R, Meador R.Inguinal lymphadenopathy as the initial presentation of sarcoidosis. Proc (Bayl Univ Med Cent). 2013;26:161-162.

2. Rybicki BA, Maliarik MJ, Major M, Popovich J Jr, Iannuzzi MC, et al. Epidemiology, demographics, and genetics of sarcoidosis. Semin Respir Infect. 1998; 13:166-173.

3. Siltzbach LE, James DG, Neville E, Turiaf J, Battesti JP, Sharma OP, Hosoda Y, Mikami R, Odaka M. Course and prognosis of sarcoidosis around the world. Am J Med. 1974;57:847-852.

4. Statement on sarcoidosis. Joint Statement of the
American Thoracic Society (ATS), the European Respiratory Society (ERS) and the World Association of Sarcoidosis and Other Granulomatous Disorders (WASOG) adopted by the ATS Board of Directors and by the ERS Executive Committee. ATS/ERS/WASOG Committee Statement on sarcoidosis. Am J Respir Crit Care Med. 1999;160:736-755.

5. Nunes H, Soler P, Valeyre D. Pulmonary sarcoidosis. Allergy. 2005;60:565-558.

6. De Vries J, Wirnsberger RM. Fatigue, quality of life and health status in sarcoidosis. Eur Respir Monograph. 2005;10:92-104.

7. Nunes H, Brillet PY, Valeyre D, Brauner MW, Wells AU. Imaging in sarcoidosis. Sem Respir Crit Care Med. 2007;28:102-120.

8. Hillerdal G, Nou E, Osterman K, Schmekel B, et al. Sarcoidosis: epidemiology and prognosis. A 15-year European study. Am Rev Respir Dis. 1984;130:29-32.

9. Rybicki BA, Iannuzzi MC. Epidemiology of Sarcoidosis: Recent Advances and Future Prospects. Seminars in Respiratory and Critical Care Medicine, 2007;20:22-35.

10. Grutters JC, van den Bosch JM. Corticosteroid treatment in sarcoidosis. Eur Respir J. 2006;28:627-636.

11. Baughman RP, Teirstein AS, Judson MA, Rossman MD, Yeager H, Jr, Bresnitz EA, et al. for Case Control Etiologic Study of Sarcoidosis (ACCESS) research group. Clinical characteristics of patients in a case control study of sarcoidosis. Am J Respir Crit Care Med. 2001;164:1885-1889. 